\title{
Carpal Tunnel Syndrome Caused by Bifid Median Nerve in Association with Anomalous Course of the Flexor Digitorum Superficialis Muscle at the Wrist
}

\author{
Jong-Jin Park', Jin-Gyu Choi ${ }^{1}$, Byung-Chul Son ${ }^{1,2}$ \\ ${ }^{I}$ Department of Neurosurgery, Seoul St. Mary's Hospital, College of Medicine, The Catholic University of Korea, Seoul, \\ ${ }^{2}$ Catholic Neuroscience Institute, College of Medicine, The Catholic University of Korea, Seoul, Korea
}

\begin{abstract}
Secondary carpal tunnel syndrome (CTS) can be caused by vascular anomalies usually involving persistent median artery, variations of the median nerve, and space-occupying lesions in the wrist and palm. High division of the median nerve proximal to the carpal tunnel (known as a bifid median nerve) is a median nerve anomaly with a reported incidence of $2.8 \%$ per wrist. The bifid median nerve is often associated with various abnormalities such as persistent median artery and aberrant muscles, causing clinical features of CTS. Most reported cases of bifid median nerves are associated with CTS due to its higher cross-sectional area compared to a non-bifid median nerve. We report a rare case in which an anomalous tendinous course of the flexor digitorum superficialis muscle is associated with bifid median nerve proximal to the flexor retinaculum at the distal wrist. Surgeons should consider the possibility of median nerve variation in patients with unilateral severe CTS and be aware of this anomaly during elective carpal tunnel release.
\end{abstract}

Key Words: Carpal tunnel syndrome $\cdot$ Congenital abnormalities $\cdot$ Median nerve $\cdot$ Median neuropathy $\cdot$ Tendons

\section{INTRODUCTION}

Carpal tunnel syndrome (CTS) is a common neuropathy caused by entrapment of the median nerve by a thickened flexor retinaculum in the wrist ${ }^{8)}$. Median nerve entrapment at the wrist is rarely associated with anatomical variations of the median nerve and anomalous muscles ${ }^{1,2)}$. Variations in distal median nerve included aberrations of the median nerve itself and its motor as well as palmar cutaneous branches ${ }^{1-4,6,7,9,10)}$. Anomalies of the muscles and tendons ${ }^{1-4,6,7)}$ and persistence of the median artery ${ }^{4}$ have also been noted within the carpal tunnel both proximally and distally. Although uncommon, these anatomical variations have been reported to be associated with failure of open carpal tunnel surgery and risks of median nerve injury ${ }^{1,7}$. They should be anticipated during surgery ${ }^{1)}$.

Received: December 7, 2016, Revised: December 22, 2016 Accepted: January 26, 2017

Corresponding author: Byung-chul Son, MD, PhD

Department of Neurosurgery, Seoul St. Mary's Hospital, College of

Medicine, The Catholic University of Korea, 222 Banpo-daero,

Seocho-gu, Seoul 06591, Korea

Tel: +82-2-2258-6122, Fax: +82-2-594-4248

E-mail: sbc@catholic.ac.kr

\section{CASE REPORT}

A 55-year-old right-handed female patient presented with a 3-year history of progressively worsening paresthesias, numbness, and tingling in the lateral three digits and radial palm in her right hand. There was a constant aching pain in the radial palm. There was no neck pain or radicular symptom in her arms. Her medical history was unremarkable. Physical examination showed thenar muscle atrophy and muscle weakness of the abductor pollicis brevis in the right hand. Tendon reflexes were normal and symmetric. Decreased sensation to light touch and pinprick was evident in the lateral three digits of the right hand. Tinel sign was not elicited. However, a positive Phalen test was present on the right wrist. Pain and paresthesia in the right hand initially responded to repeated local steroid injection and medications. The symptoms of the patient progressively worsened over time, eventually showed no response to conservative treatment 6 months prior to admission.

Electrodiagnostic findings were compatible with median entrapment neuropathy around the right wrist and clinical carpal tunnel syndrome with severe degree by American Association of Electrodiagnostic Medicine (AAEM) classification ${ }^{8)}$. Considering her unusually severe pain associated with typical features of carpal tunnel syndrome and unilateral involvement, 
an magnetic resonance imaging (MRI) was requested. MRI revealed a bilobed appearance of partially bifid median nerve in distal forearm proximal to the flexor retinaculum (Fig. 1A). The median nerve in the carpal tunnel at the level of the pisiform bone was enlarged and the median nerve cross-section area was $16.02 \mathrm{~mm}^{2}$ (ImageJ, NIH) (Fig. 1B). There was no abnormal signal intensity or gadolinium enhancement along the course of the median nerve in the MRI.

After incision of the skin and superficial fascia of the distal forearm and the palm, the underlying flexor retinaculum was identified and transected. The forearm fascia was then carefully dissected. The underlying median nerve just proximal to the flexor retinaculum in distal forearm was found to be duplicated. A tendon of the flexor digitorum superficialis (FDS) muscle traversed between the radial and ulnar division of the bifid median nerve (Fig. 1C). There was no median artery. After dissection of the subparaneurial space, the adhesion between the tendon of the FDS and the bifid median nerve was detached carefully. The median nerve was mobilized free from compression by the FDS tendon (Fig 1D).

Severe pain in radial palm and paresthesia disappeared immediately after the operation. Hand weakness improved gradually over 2 weeks. However, hypesthesia in the distal portion of the radial three digits remained until 12-month follow-up at an outpatient clinic.

\section{DISCUSSION}

High bifurcation of the median nerve, the so-called bifid median nerve, is an well-known but rare anomaly of the median nerve. Variations of the median nerve were first classified by Lanz ${ }^{7}$. Group I includes variations of the course of the thenar branch. Group II includes accessory branches at the distal carpal tunnel. Group III includes those with a high division of the nerve. Group 4 includes accessory branches proximal to the carpal tunnel. According to this classification ${ }^{7}$, bifid median nerve is mentioned as a group 3 variation. The incidence of high bifurcation of the median nerve was reported to be $2.8 \%$ in the series by Lanz $^{7}$ and $3.3 \%$ by Amadio ${ }^{2}$. However, its prevalence has increased from $2 \%$ to $26 \%$ per wrist by ultrasound examinations ${ }^{3)}$. It seems that the wide variations in the prevalence of this anomaly stem from the fact that the results of earlier reports ${ }^{1,2,9,10)}$ are from intraoperative findings during carpal tunnel release and repair of wrist laceration $^{1)}$.

The two branches of the nerve which run parallel inside the tunnel are sometimes separated by a persistent median artery or an accessory muscle ${ }^{7}$. Sometimes one of the two branches might appear in an accessory compartment situated under the retinaculum of the flexor tendons. Lanz ${ }^{7)}$ has described that the two parts of the nerve are usually equal in size. However, Schultz et al. ${ }^{9)}$ have noted that the two parts may be unequal in size, with the larger radial division being approximately equal in size to a normal median nerve. Bifid median nerves have been reported to be independent risk factors for the development of carpal tunnel syndrome because they tend to have a relatively higher cross-sectional area than non-bifid median nerves and occupy more room in the carpal tunnel ${ }^{3)}$. In the current case, two parts of the median nerve were found to be equally swollen intraoperatively and crosssection area at the level of distal wrist in preoperative MRI were measured to be 6.1 and $5.8 \mathrm{~mm}^{2}$ (radial and ulnar divisions), respectively. However, cross-sectional area of the median nerve at the level of the pisiform was $16 \mathrm{~mm}^{2}$, which was much greater than an average cutoff value of $9-10 \mathrm{~mm}^{2}$ for carpal tunnel syndrome in nonbifid median nerves at the level of the pisiform ${ }^{2,3)}$.

The occurrence of a bifid median nerve has been reported to be frequently associated with persistent median artery ${ }^{1,2,4,6,7}$. The median artery is the dominant blood supply to the hand in utero. It normally regresses after the embryonic stage (50 days gestation) ${ }^{1)}$. When it persists, it may supply the superficial palmar arch or may be the main blood supply to the radial two digits ${ }^{1)}$. Anatomical studies have reported that the prevalence of the median artery is in $10 \%$ to $20 \%$ of cadaveric dissection ${ }^{2)}$. Although a persistent median artery can cause carpal tunnel syndrome, it is unnecessary to ligate or resect the artery. Simple carpal tunnel release could relieve the symptoms ${ }^{1}$.

Aberrant muscles associated with bifid median nerve have been sporadically reported to cause carpal tunnel syndrome ${ }^{3,6,7,9)}$. Embryologically, the presence of anomalous muscle is thought to be the cause of bifidity ${ }^{1}$. Until now, four types of anomalous muscles have been reported: an accessory first lumbrical, an accessory palmaris longus, a prolonged flexor superficialis muscle belly, and a palmaris profundus. Most patients with anomalous muscles associated with bifid median nerves presented with carpal tunnel syndrome. Failure of excision of the anomalous muscle at the time of carpal tunnel release has resulted in persistent symptoms ${ }^{1,10)}$. In the current case, we dissected the radial and ulnar divisions of the bifid median nerve from tendinous adhesion to aberrant flexor digitorum superficialis muscle and symptoms were relieved without recurrence.

It is difficult to suspect an anomaly of median nerve in every case of carpal tunnel syndrome preoperatively. However, considering the incidence of variations of the median nerve, surgeons should keep in mind potential surgical hazards and recurrence of symptoms with insufficient surgical decompression. It has been suggested that MRI and ultrasound examinations can be performed to show bifid median nerves 
so that surgeons can avoid potential surgical risks ${ }^{1-3)}$.

Although we do not routinely use MRI or other imaging modalities for the diagnosis of carpal tunnel syndrome, we always perform ultrasound examination and high-resolution MRI in every suspicious case of secondary carpal tunnel syndrome. It has been suggested that imaging studies should be obtained for patients in whom secondary carpal tunnel syndrome is suspected with severe unilateral symptoms, young age ( $<40$ years), and male sex ${ }^{2}$. In the current case, we perfomed an MRI study for unexplained and severe unilateral symptoms of three-year duration and high bifurcation of the median nerve was identified prior to the operation. Although carpal tunnel syndrome presents bilaterally in 59\% to $87 \%$ of patients ${ }^{2,10)}$, bilateral neurophysiological impairment with later contralateral involvement in the follow-up has been suggested $^{2}$. The incidence of space-occupying lesions in unilateral carpal tunnel syndrome is also higher than that of bilateral carpal tunnel syndrome ${ }^{2,10}$.

When this anatomical variation is present, it is important to have carpal tunnel release because of the increased risk of nerve injury. In addition, the two branches of the nerve might be constricted separately. Separate decompression might be required for each branch ${ }^{10)}$. Furthermore, branches of the

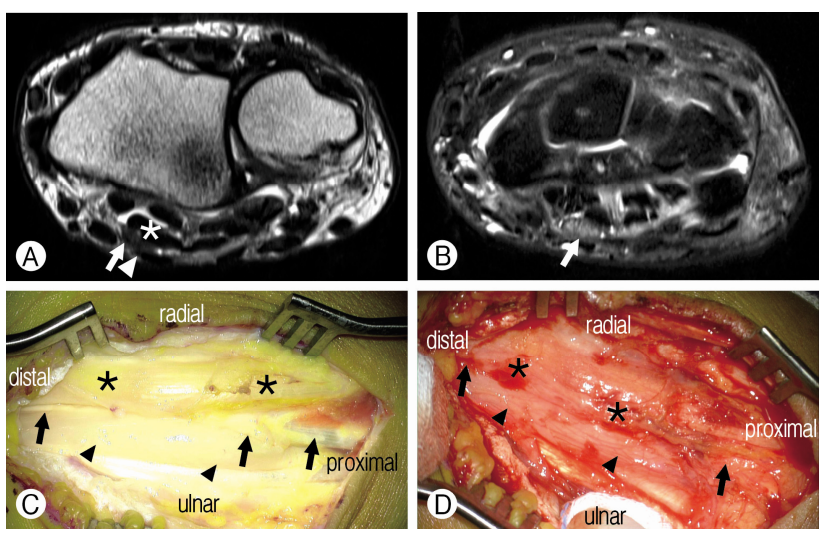

Fig. 1. (A) An axial, T2-weighted magnetic resonance imaging (MRI) of the right wrist showing bilobed median nerve just proximal to the flexor retinaculum in the distal forearm. Note the radial (arrow) and ulnar (arrowhead) divisions of the median nerve and adherent flexor digitorum superficialis muscle (asterisk). (B) A T2-weighted axial, fat suppression MRI image of the swollen median nerve (arrow) within carpal tunnel at the level of the pisiform. (C) An intraoperative photography showing the radial (black asterisks) and ulnar (black arrowheads) divisions of the bifid median nerve and the course of aberrant flexor digitorum profundus muscle (black arrows) in the distal forearm proximal to the flexor retinaculum. (D) An intraoperative photography showing the radial (black asterisks) and ulnar (black arrowheads) divisions of the bifid median nerve in the distal forearm after neurolysis and decompression of the aberrant muscle (black arrows). bifid median nerve might be confused with flexor tendons with a risk for an inadvertent injury ${ }^{10)}$. Although bifid median nerve is a rare anatomic variation, surgeons attempting carpal tunnel release should be aware of it to avoid possible surgical hazard.

\section{CONCLUSION}

We report a rare case of carpal tunnel syndrome with bifid median nerve in association with aberrant flexor digitorum superficialis muscle. It was not associated with persistent median artery. The present case highlights the importance of clear visualization of the median nerve and surrounding structures during surgical treatment of carpal tunnel release and knowledge of anatomical variation of the median nerve. Inadvertent injury to the median nerve during carpal tunnel surgery can be minimized if these variations are recognized prior to operation.

\section{CONFLICTS OF INTEREST}

No potential conflict of interest relevant to this article was reported.

\section{REFERENCES}

1. Al-Qattan MM, Al-Zahrani K, Al-Omawi M: The bifid median nerve re-visited. J Hand Surg (Eur) 34E:212-214, 2009

2. Amadio PC: Anatomical variations of the median nerve within carpal tunnel. Clin Anat 1:23-31, 1988

3. Bayrak IK, Bayrak AO, Kale M, Turker H, Diren B: Bifid median nerve in patients with carpal tunnel syndrome. J Ultrasound Med 27:1129-1136, 2008

4. Fernadez-Garcia S, Pi-Folguera J, Estallo-Matino F: Bifid median nerve compression due to a musculotendinous anomaly of FDS to the middle finger. J Hand Surg (Br) 19B:616-617, 1994

5. Jang S, Choi J, Son B: Compression of the median neve by a lipoma in the distal forearm associated with bilateral carpal tunnel syndromes. The Nerve 2:84-86, 2016

6. Kornberg M, Aulicino PL, DePuy TE: Bifid median nerve within three thenar branches, case report. J Hand Surg 8:583-584, 1983

7. Lanz U: Anatomical variations of the median nerve in the carpal tunnel. J Hand Surg 2:44-53, 1977

8. Preston DC, Shapiro BE: Median neuropathy at the wrist. In: Electromyography and Neuromuscular Disorders. 2nd ed. Philadelphia, PA: Elsevier Butterworth-Heinemann; 2005:255-279.

9. Schultz RJ, Endler PM, Huddleston HD: Anomalous median nerve and anomalous muscle belly of the first lumbrical associated with carpal tunnel syndrome. J Bone Joint Surg 55A: 1733-1746, 1973

10. Yildirim A, Akan M, Aydoğdu E: Bifid median nerve. Plast Reconstr Surg 108:584-585, 2001 